\title{
Properties of the power envelope for tests against both stationary and explosive alternatives: the effect of trends
}

\author{
Patrick Marsh* \\ School of Economics and Granger Centre for Time Series Econometrics \\ University of Nottingham \\ University Park
}

NG7 2RD

email: patrick.marsh@nottingham.ac.uk

February 11th 2019

\begin{abstract}
This paper details a precise analytic effect that inclusion of a linear trend has on the power of Neyman-Pearson point optimal unit root tests and thence the power envelope. Both stationary and explosive alternatives are considered. The envelope can be characterized by probabilities for two, related, sums of chi-square random variables. A stochastic expansion, in powers of the local-tounity parameter, of the difference between these loses its leading term when a linear trend is included. This implies that the power envelope converges to size at a faster rate, which can then be exploited to prove that the power envelope must necessarily be lower. This effect is shown to be, analytically, greater asymptotically than in small samples and numerically far greater for explosive than for stationary alternatives. Only a linear trend has a specific rate effect on the power envelope, however other deterministic variables will have some effect. The methods of the paper lead to a simple direct measure of this effect which is then informative about power, in practice.
\end{abstract}

*Thanks are due to Francesco Bravo, Giovanni Forchini, David Harvey, Steve Leybourne, Peter Phillips, Robert Taylor and participants at seminars at the Universities of Birmingham, Manchester, Monash and York. 


\section{Introduction}

The power envelope is a fundamental measure of how effectively we can discriminate between false null hypotheses and specified alternatives. Every new unit root test, whether testing against stationary or explosive/bubble alternatives, must have its power characteristics compared with this envelope. Despite this, the analytic properties of the unit root power envelope are generally unknown. The focus has instead been on the stochastic properties of tests and estimators, capitalizing on the pioneering methods of Phillips (1987a, 1987b) and Chan and Wei (1987).

This paper seeks to capture the precise effect, on the power envelope, of the inclusion of a linear trend. For Economic data the unit root remains one of the most tested hypotheses. And the inclusion or otherwise of a linear trend has a profound effect on both the theoretical and observed properties of unit root tests, see both Elliott, Rothenberg and Stock (1996) and Nielsen (2008). To emphasize the importance of this, as measured via numerical resolution of the asymptotic power envelope of the former paper and in the context of an autoregressive parameter $\rho_{T}=1+c / T$, tests can have $50 \%$ power against a local alternative value $c=-7$ with no linear trend, but not until $c=-13.5$ if there is. The net effect of a linear trend on power is equivalent to a practitioner discarding $48 \%$ of their data. There is no other context in Econometrics where the effect of a single regressor is so profound.

Since there is no uniformly best invariant (UBI) test against either stationary or explosive alternatives the power envelope is constructed via the union of the powers of the continuum of point optimal tests. For each, a critical value is first required to fix size under the null, before its power is evaluated under the alternative. Therefore, for every value under the alternative two probabilities must be considered. In this paper these are characterized via probabilities for two, related, weighted sums of chisquared random variables, similar to the original representations in Dickey and Fuller (1979). These two weighted sums generally have a stochastic difference, near $c=0$, of order $O_{p}\left(c^{2}\right)$. When there is a linear trend, this falls to $O_{p}\left(c^{4}\right)$, asymptotically.

This induces a change in the rate of convergence of the power envelope itself to the chosen size. Specifically, for arbitrarily small positive $\varpi_{1}$ and $\varpi_{2}$ it is $O\left(c^{2-\varpi_{1}}\right)$ when there is no linear trend and $O\left(c^{4-\varpi_{2}}\right)$, when there is. This step change can be exploited to formally prove that powers of linear trend invariant tests are necessarily 
lower. Intuitively this arises because the covariance and its derivative are proportional, when there is a unit root. The algebraic mechanism by which this occurs can also be used to construct a simple measure of the impact of regressor invariance on any hypothesis on the covariance structure of data. In the current context this measure correlates very well with power.

The next Section presents the main results, two Lemmas (proved in the online supplementary material to this paper) and a Theorem detailing the analytic effects of a trend which is proved in the Appendix. Section 3 discusses the implications of these results utilizing numerical results also presented in tables in the supplementary material.

\section{Characterization of the Power Envelope and its Properties}

The Gaussian power envelope is constructed from the powers of each point optimal test, e.g. see King (1980) and King and Sriananthakumar (2015). However, as is clear from Elliott, Rothenberg and Stock (1996) and Marsh (2011), the asymptotic distribution of these tests is the same under far more general assumptions. Let $\left(y_{t}\right)_{t=1}^{T}$ be generated from,

$$
y_{t}=d_{t}+u_{t} \quad: \quad d_{t}=x_{t}^{\prime} \beta \quad \text { and } \quad u_{t}=\rho_{T} u_{t-1}+\varepsilon_{t},
$$

where $x_{t}$ is a $k \times 1$ deterministic regressor, $\beta$ a $k \times 1$ unknown parameter, $\varepsilon_{t}$ is a zero mean error process and we put $\rho_{T}=1+c / T$.

We will consider tests of $H_{0}: c=0$ against both stationary (S) and explosive (E) alternatives, as in

$$
H_{1}^{S}: c<0 \text { and } H_{1}^{E}: c>0 .
$$

Elliot, Rothenberg and Stock (1996), under their Condition A, provide representations of the power envelope against $H_{1}^{S}$ in two cases. First when $d_{T}=o\left(T^{1 / 2}\right)$ (their equation (4)) and second when $d_{t}=\beta_{1}+\beta_{2} t$ (their equation (8)). Here we will denote those two, size $\alpha$, envelopes by $\Pi_{\alpha}^{\mu}(c)$ and $\Pi_{\alpha}^{\tau}(c)$, respectively. Full expressions for these are also provided in the supplementary material. Although originally provided only for tests against $H_{1}^{S}$, power envelopes for $H_{1}^{E}$ can also be generated using the 
results of Phillips (1987b) and Chan and Wei (1987), see for example Harvey and Leybourne (2014).

Let $y=\left(y_{1}, . ., y_{T}\right)^{\prime}, X=\left(x_{1}, . ., x_{T}\right)^{\prime}, \beta=\left(\beta_{1}, . ., \beta_{k}\right)^{\prime}, L$ be a lower triangular matrix with $1^{\prime} s$ on the first lower diagonal and $0^{\prime} s$ elsewhere, $\Delta_{\rho}=I-\rho L$ and let $W=\Delta_{1} X$. Put $n=T-k$, and define

$$
A=A_{c}=C^{\prime} \Delta_{1}\left(\Delta_{1+c / T}^{\prime} \Delta_{1+c / T}\right)^{-1} \Delta_{1}^{\prime} C
$$

where $C C^{\prime}=M_{W}=I-W\left(W^{\prime} W^{-1}\right) W^{\prime}$ and $C^{\prime} C=I_{n}$, and let $\lambda_{i}, i=1, . ., n$, be the ordered eigenvalues of $A$. Finally let $z=\left(z_{1}, . ., z_{n}\right)^{\prime}=C^{\prime} \Delta_{1} y$ and define the following two statistics;

$$
Q_{0, n}(c)=\sum_{i=1}^{n} \lambda_{i}^{-1} \bar{z}_{i}^{2} \text { and } Q_{1, n}(c)=\left(\sum_{i=1}^{n} \lambda_{i} \bar{z}_{i}^{2}\right)^{-1},
$$

where $\bar{z}_{i}=z_{i} / \sqrt{\sum_{i=1}^{n} z_{i}^{2}}$.

The following Lemma provides alternative characterizations of the asymptotic power envelopes as well as a stochastic expansion of the limits of the two statistics defined in (4). Both the general assumptions under which it applies and its algebraic demonstration is given in the supplementary material.

Lemma 1 (i) Let $\left\{\tilde{z}_{i}\right\}_{i \in \mathbb{Z}}$ denote a sequence of IID $N(0,1)$ random variables, then the asymptotic power envelope at size $\alpha$ for testing either $H_{1}^{S}$ and $H_{1}^{E}$, for any set of explanatory variables $X$, can be characterized by

$$
\Pi_{\alpha}(c)=\operatorname{Pr}\left[Q_{1}(c)<\kappa_{\alpha}\right], \quad Q_{1}(c)=\lim _{n \rightarrow \infty}\left(\sum_{i=1}^{n} \lambda_{i} \tilde{z}_{i}^{2}\right)^{-1},
$$

where the critical value $\kappa_{\alpha}$ is defined by

$$
\alpha=\operatorname{Pr}\left[Q_{0}(c)<\kappa_{\alpha}\right], \quad Q_{0}(c)=\lim _{n \rightarrow \infty} \sum_{i=1}^{n} \lambda_{i}^{-1} \tilde{z}_{i}^{2} .
$$

(ii) Denote the $j^{\text {th }}$ derivative of $A$ with respect to $c$, evaluated at 0 , by $D_{j}$, and let $\left\{\delta_{1, i}\right\}_{1}^{\infty},\left\{\delta_{2, i}\right\}_{1}^{\infty}$ and $\left\{\delta_{12, i}\right\}_{1}^{\infty}$ be the eigenvalues of the matrices $D_{1}, D_{2}$ and $D_{1} D_{2}$, and let $\left\{\xi_{1, i}^{2}\right\}_{1}^{\infty},\left\{\xi_{2, i}^{2}\right\}_{1}^{\infty}$ and $\left\{\xi_{12, i}^{2}\right\}_{1}^{\infty}$ be sequences of independent chi-square variables, then in a neighbourhood of $c=0$,

$$
Q_{1}(c)-Q_{0}(c)=c^{2} \gamma_{1}+\frac{c^{3}}{6} \gamma_{2}+O_{p}\left(c^{4}\right)
$$


where $\gamma_{1}$ and $\gamma_{2}$ are defined by

$$
\gamma_{1}=\lim _{n \rightarrow \infty}\left\{\left(\frac{1}{n} \sum_{i=1}^{n} \delta_{1, i} \xi_{1, i}^{2}\right)^{2}-\frac{1}{n} \sum_{i=1}^{n} \delta_{1, i}^{2} \xi_{1, i}^{2}\right\},
$$

and

$$
\begin{aligned}
\gamma_{2}= & \lim _{n \rightarrow \infty}\left\{3\left(\frac{1}{n} \sum_{i=1}^{n} \delta_{1, i} \xi_{1, i}^{2}\right)\left(\frac{1}{n} \sum_{i=1}^{n} \delta_{2, i} \xi_{2, i}^{2}\right)\right. \\
& -6\left(\left(\frac{1}{n} \sum_{i=1}^{n} \delta_{1, i} \xi_{1, i}^{2}\right)^{3}-\frac{1}{n} \sum_{i=1}^{n} \delta_{12, i} \xi_{12, i}^{2}+\frac{1}{n} \sum_{i=1}^{n} \delta_{1, i}^{3} \xi_{1, i}^{2}\right) .
\end{aligned}
$$

Lemma 1 provides a representation for the asymptotic power envelope in terms of an (infinite) weighted sum of chi-square random variables. Given that there is no UBI test, the properties of the power envelope can only be explored by directly comparing $Q_{1}(c)$ with $Q_{0}(c)$. Via the stochastic expansions presented in Lemma 1 (ii) we can establish the rate of convergence of the asymptotic power envelope to the chosen size, as in the following Lemma.

Lemma 2 Let $\bar{Q}=c^{-2 k}\left(Q_{0}(c)-Q_{1}(c)\right)$, where $k$ is such that $\bar{Q}$ is $O_{p}(1)$, then the power envelope satisfies

$$
\Pi_{\alpha}(c)=\alpha+O\left(c^{2 k-\varpi_{k}}\right)
$$

where $\varpi_{k}>0$ is an arbitrarily small constant.

Generally $Q_{1}(c)-Q_{0}(c)=O_{p}\left(c^{2}\right)$ and so the envelope is locally $\alpha+O\left(c^{2-\varpi_{1}}\right)$. Now denote the column space of $X$ by $\mathcal{M}(X)$ and the linear trend by $\tau=(t)_{t=1}^{T}$. Suppose now a linear trend is included in the regressors, i.e. $\tau \in \mathcal{M}(X)$, then the following Theorem, proved in the Appendix, demonstrates that in this case $Q_{1}(c)-$ $Q_{0}(c)=O_{p}\left(c^{4}\right)$, and thus the envelope is $\alpha+O\left(c^{4-\varpi_{2}}\right)$. As with Moon, Perron and Phillips (2007) the effect manifests itself as an order of magnitude step change in the order of convergence, although here in the parameter itself. These results hold in a neighbourhood of $c=0$, however by exploiting the analytic properties of $\Pi_{\alpha}(c)$ these findings can be continued to demonstrate that inclusion of a linear trend necessarily implies the power envelope is strictly lower for any finite value of $c$. 
Theorem 1 i) Suppose that $\tau \in M(X)$, then

$$
Q_{1}(c)-Q_{0}(c)=O_{p}\left(c^{4}\right)
$$

ii) At the a significance level,

$$
\begin{aligned}
& \text { if } \tau \notin \mathcal{M}(X) \text { then } \Pi_{\alpha}(c)-\alpha=O\left(c^{2-\varpi_{1}}\right) \text {, while } \\
& \text { if } \tau \in \mathcal{M}(X) \text { then } \Pi_{\alpha}(c)-\alpha=O\left(c^{4-\varpi_{2}}\right) .
\end{aligned}
$$

iii) Let the set of regressors $X$ satisfy $x_{T}^{\prime} \beta=o\left(T^{1 / 2}\right)$, so that the power envelope is $\Pi_{\alpha}^{\mu}(c)$. If we add the column $\tau$ to $X$, then we obtain power envelope $\Pi_{\alpha}^{\tau}(c)$, which satisfies

$$
\Pi_{\alpha}^{\tau}(c)<\Pi_{\alpha}^{\mu}(c)
$$

for all finite $c$.

\section{Analysis and Conclusions}

i) Parts (i) and (ii) of Theorem 1 apply only in a neighbourhood of $c=0$. However, the power envelope (via (5) and (6)) is a function of both regressor set $X$ and local parameter $c$ through the eigenvalues of the matrix $A_{c}$. Since these eigenvalues are analytic in $c$, then so is the power envelope. That $\Pi_{\alpha}^{\tau}(c)$ is smaller than $\Pi_{\alpha}^{\tau}(c)$ at some, local, value of $c$ can therefore be analytically continued to all finite values of c. This finding links directly to findings in Nabeya and Tanaka (1990), which shows that there is no Locally Best Invariant test of a unit root when there is a linear trend although that paper contains no explicit results for the power envelope, itself. Equally Theorem 1 explains how the precise finding of Marsh (2007a) for the null $c=0$ has, in fact, an impact for any finite value of $c$ under the alternative. In the absence of any formal distribution theory, asymptotic or otherwise, for trend invariant estimators or tests, Theorem 1 offers the only analytic demonstration of the power loss of such trends, hitherto observed only experimentally.

ii) The local expansions remain informative about the power envelope globally. Table 1a in the supplementary material presents outcomes of the power envelope for 
a variety of simple choices of $d_{t}$ (a constant, a linear trend and trends involving the logarithm, square root, square and exponent of time) in (1) for $T=250$. It is worth noting that not all trends are associated with low power, exponential trends imply powers similar to those of the constant case. In Table $1 \mathrm{~b}$ the power envelopes are approximated using stochastic expansions of $Q_{0, n}(c)$ and $Q_{1, n}(c)$ to order $O_{p}\left(c^{3}\right)$. As is evident comparing across entries in Tables $1 \mathrm{a}$ and $1 \mathrm{~b}$ simulation of just the leading terms of these statistics capture the envelope almost entirely.

iii) Although the results in Theorem 1 are asymptotic, their proof yields the insight that the effect of a linear trend can be greater asymptotically, than in finite samples. From the proof of Theorem 1(i), when there is a trend, the $O_{p}\left(c^{3}\right)$ term in the stochastic difference between $Q_{0, n}(c)$ and $Q_{1, n}(c)$ is

$$
\frac{c^{3}}{2 T}\left[\left(\sum_{i=1}^{n} \delta_{2, i} \tilde{\xi}_{2, i}^{2}-2 \sum_{i=1}^{n} \delta_{2, i} \tilde{\xi}_{12, i}^{2}\right)\right]
$$

which converges in probability to 0 only as $T \rightarrow \infty$. This indicates a differential relative effect that a linear trend has on the finite sample and asymptotic envelopes. To illustrate, Table 2 contains the ratios of the power envelopes evaluated for $d_{t}=\beta_{1}+\beta_{2} t$ and $d_{t}=\beta_{1}$ for values of $c$ from 1.25 to -5.0 and for different significance levels, $\alpha=.01, .05, .10$ evaluated for sample sizes of $T=50,250,500$. The effects are clear and significant, particularly when $c$ is small. This difference in the behaviour of the asymptotic and finite sample envelopes has significance for the choice of unit root tests in practice. As Francke and de Vos (2007) note, tests designed to have power close to the asymptotic power envelope may not have power functions close to the finite sample one, in the presence of trends. This can only be explained via the quantitative difference between them found in this paper. It is also suggestive that new tests ought to be compared to both finite sample and asymptotic envelopes to justify their properties.

iv) The mechanism by which the power envelope is reduced on inclusion of a linear trend is algebraic. Specifically, as in the proof of Theorem 1(i), letting $\Sigma_{1+c / T}=$ $\left(\Delta_{1+c / T}^{\prime} \Delta_{1+c / T}\right)^{-1}$ be the covariance of a pure 'near unit root' process, then

$$
\left.\frac{d \Sigma_{1+c / T}}{d c}\right|_{c=0}=-\left(\Delta_{1}^{-1}\left(\Delta_{1}^{-1}\right)^{\prime}-\tau \tau^{\prime}\right)
$$

To construct invariant tests we first let $w=C^{\prime} \Delta_{1} y$, which removes dependence on $\beta$. Let $V[w]=\sigma^{2} A_{c}$ so that the null implies $H_{0}: V[w]=\sigma^{2} A_{0} \propto I_{n}$. If we take a series 
expansion of $A_{c}$ around $0, A_{c}=A_{0}+\sigma^{2} \sum_{j=1}^{\infty} \frac{c^{j}}{j !} D_{j}$, where $D_{j}=d^{j} A_{c} /\left.d c^{j}\right|_{c=0}$ then when $X$ contains a linear trend we find $D_{1}=-T^{-1} I_{n}=-T^{-1} A_{0}$ and hence

$$
\sigma^{2} A_{c}=\sigma^{2}\left(1-c T^{-1}\right) A_{0}+\sigma^{2} \sum_{j=2}^{\infty} \frac{c^{j}}{j !} D_{j}
$$

That is $\sigma^{2} A_{c}$ is proportional to $\sigma^{2} A_{0}$ up to and including the $O(c)$ term when there is a linear trend. Since we also require scale invariance this, heuristically, captures the effective cause of the dramatic loss of power. Algebraically this proportionality is exact in the unit root/linear trend problem. Generically, suppose we wish to test $H_{0}: V[w]=\sigma^{2} A_{0}$ vs. $H_{1}: V[w]=\sigma^{2} A_{c}$. If the derivative of $A_{c}$ at $c=0$ is $D_{1}$ then we would expect low power if $A_{0}$ and $D_{1}$ are proportional. A simple measure of the proportionality of two matrices is the variation in the ratio of their respective ordered eigenvalues, $\lambda_{i}$ and $\delta_{1, i}$. To proceed, let $\bar{\lambda}_{X}=n^{-1} \sum_{i=1}^{n} \lambda_{i} / \delta_{1, i}$ and $S_{X}^{2}=\sum_{i=1}^{n}\left(\frac{\lambda_{i}}{\delta_{1, i}}-\bar{\lambda}_{X}\right)^{2}$, and define

$$
\Lambda_{X}^{2}=S_{X}^{2} / S_{\varnothing}^{2}
$$

where $\varnothing$ denotes the null set, i.e. $X$ is empty and no invariance is required in the construction of $w \cdot \Lambda_{X}^{2}$ thus measures the relative variation in eigenvalues for a given choice of $X$ compared to the case of no regressors, i.e. only scale invariance is required. In the linear trend case $\bar{\Lambda}_{X}^{2}=0$. For example, in the cases enumerated in the supplementary material we find, with $T=250$,

$$
\begin{array}{l|llllll}
d_{t} & \beta_{1} & \beta_{1}+\beta_{2} t & \beta_{1}+\beta_{2} \ln (t) & \beta_{1}+\beta_{2} \sqrt{t} & \beta_{1}+\beta_{2} t^{2} & \beta_{1}+\beta_{1} e^{t} \\
\hline \bar{\Lambda}_{X}^{2} & 0.996 & 0.000 & 0.759 & 0.126 & 0.058 & 0.979
\end{array} .
$$

In terms of ranking these outcomes match perfectly the power envelopes given in Table 1a. This measure could be adapted for any (simple) hypothesis test on a covariance matrix, when invariance with respect to the mean is required. It provides a simple measure of the sensitivity of power to the choice of deterministics, similar in spirit to the analysis of Leamer (1985).

v) Bykhovskaya and Phillips (2018) explore tests involving functional local alternatives where the local parameter depends on time. e.g. $H_{0}: c_{t}=0$ vs. $H_{1}^{F}: c_{t}=$ $c(t / T) / T$, so that only the initial value has a unit root. Although invariance with respect to neither the mean nor scale is pursued in that paper, it is trivial to apply 
the framework here to such cases, as well as to functional stationary alternatives, where $c<0$. Define $\tilde{\Delta}_{c}=I_{T}-L\left(I_{T}+c / T \operatorname{diag}\{t / T\}_{t=1}^{T}\right)$ then the error covariance matrix under $H_{1}^{F}$ is $\tilde{\Sigma}_{c}=\left(\tilde{\Delta}_{c} \tilde{\Delta}_{c}^{\prime}\right)^{-1}$ and its derivative at $c=0$ is the $T \times T$ matrix, $\tilde{\Sigma}_{0}^{1}$ having $(i, j)^{t h}$ element $\max [i, j]-i \mathbb{I}(i=j)$, where $\mathbb{I}($.$) is the indicator$ function. In this case the covariance of $w=C^{\prime} \Delta_{1} y$ is $\tilde{A}_{c}=C^{\prime} \Delta_{1} \tilde{\Sigma}_{c} \Delta_{1}^{\prime} C$ and has slope $\tilde{D}_{1}=C^{\prime} \Delta_{1} \tilde{\Sigma}_{0}^{1} \Delta_{1}^{\prime} C$. Even in the case that $X$ contains a linear trend $\tilde{A}_{0}$ is not proportional to $D_{1}$.

Calculating the eigenvalue variation defined in (7) we find $\bar{\Lambda}_{X}^{2}=0.997$ for the constant case and $\bar{\Lambda}_{X}^{2}=0.993$ for the linear trend case, so the relative impact of a linear trend is extremely small when testing against functional alternatives. This is bourne out in the outcomes for the ratios of the power envelopes presented in Table 3 which repeat the experiments reported in Table 2, but for the functional alternatives, $H_{1}^{F}$, in both stationary and explosive directions.

vi) The focus thus far has been on the theoretical implications on the testing problem of the inclusion of a linear trend. Harvey et al (2009) detail practical procedures which account for uncertainty over whether or not a trend is required. Numerically, their tests are shown to have power curves close to $\Pi_{\alpha}^{\mu}(c)$ when there is no trend, and close to $\Pi_{\alpha}^{\tau}(c)$ when there is. Marsh (2009) characterizes this uncertainty in terms of a Bernoulli mixture of the trend and no-trend cases. The results of this paper demonstrate, unequivocally, the necessity of the Harvey et al (2009) pre-test or union of rejections based tests. Specifically, under such uncertainty, any other test must either be inefficient (its power will be bounded by $\Pi_{\alpha}^{\tau}(c)<\Pi_{\alpha}^{\mu}(c)$ ) when no trend is present, or inconsistent when it is.

\section{Data availability statement}

Data sharing is not applicable to this article as no new data were created or analysed in this study.

\section{References}

Bykhovskaya, A. and P.C.B. Phillips 2018. Boundary Limit Theory for Functional Local to Unity Regression. J.T.S.A. 39: 523-562.

Chan, N.H. and C.Z. Wei 1987. Asymptotic inference for nearly nonstationary AR(1) processes. Annals of Statistics 15: 1050-1063. 
Dickey, D.A. and W.A. Fuller 1979. Distribution of the estimators for autoregressive time series with a unit root. J.A.S.A. 366: 427-431.

Elliott, G., Rothenberg, T.J. and J.H. Stock 1996. Efficient tests for an autoregressive unit root. Econometrica 64: 813-836.

Francke, M.K. and A.F. de Vos 2007. Marginal likelihood and unit roots. Journal of Econometrics 137: 708-728.

Harvey, D. I., Leybourne, S.J. and A.M.R. Taylor 2009. Unit Root Testing in Practice: Dealing with Uncertainty over the Trend and Initial Condition. Econometric Theory, 25: 587-636.

Harvey, D. I. and S.J. Leybourne 2014. Asymptotic behaviour of tests for a unit root against an explosive alternative. Economics Letters 122: 64-68.

King, M. L. 1980. Robust tests for spherical symmetry and their application to least squares regression. Annals of Statistics 8: 1265-1271.

King, M.L. and S. Sriananthakumar 2015. Point optimal testing: a survey of the post 1987 literature. Model Assisted Statistics and Algorithms 10: 79-196.

Leamer E.E. 1985. Sensitivity Analyses Would Help. The American Economic Review 75: $308-313$.

Marsh, P. 2007a. The available information for invariant tests of a unit root. Econometric Theory 23: 686-710.

Marsh, P. 2009. Commentaries on 'Unit Root Testing in Practice: Dealing with Uncertainty over the Trend and Initial Condition'. Econometric Theory, 25: 637657.

Marsh, P. 2011. Saddlepoint and estimated saddlepoint approximations for optimal unit root tests. Econometric Theory 27: 1026-1047.

Moon, H.R., B. Perron and P.C.B. Phillips 2007. Incidental trends and the power of panel unit root tests. Journal of Econometrics 141: 416-459.

Nabeya, S. and K. Tanaka 1990. Limiting power of unit-root tests in time-series regression. Journal of Econometrics 46: 247-271.

Nielsen, B. 2008. Power of tests for unit roots in the presence of a linear trend. Oxford Bulletin of Economics and Statistics 70: 619-644.

Phillips, P.C.B. 1987a. Time Series Regression with a Unit Root. Econometrica 55: 277-301. 
Phillips, P.C.B. 1987b. Towards a unified asymptotic theory for autoregression. Biometrika 74: 535-547.

\section{Appendix}

\section{Proof of Theorem 1}

Part (i). Suppose that $X$ contains a linear trend. Let $e=(1,1,1, . ., 1)^{\prime}$ be the constant vector, so that a linear trend is defined by $\tau=\Delta_{1}^{-1} e$, where $\Delta_{1}=I-L$. If $\mathcal{M}(X)$ is the column space of $X$, then

$$
\tau \in \mathcal{M}(X) \rightarrow \Delta_{1} \tau \in \mathcal{M}\left(\Delta_{1} X\right) \equiv \mathcal{M}(W) \Longleftrightarrow C^{\prime} \Delta_{1} \tau=0,
$$

where $C$ is defined above. The first derivative of $A_{c}$ at $c=0$ is

$$
\begin{aligned}
D_{1} & =\left.\frac{d A_{c}}{d c}\right|_{c=0}=C^{\prime} \Delta_{1}\left[\frac{d\left(\Delta_{1+c / T}^{-1}\left(\Delta_{1+c / T}^{-1}\right)^{\prime}\right)}{d c}\right]_{c=0} \Delta_{1}^{\prime} C \\
& =C^{\prime} \Delta_{1}\left[\frac{d \Delta_{1+c / T}^{-1}}{d c}\left(\Delta_{1+c / T}^{-1}\right)^{\prime}+\Delta_{1+c / T}^{-1} \frac{d\left(\Delta_{1+c / T}^{-1}\right)^{\prime}}{d c}\right]_{c=0} \Delta_{1}^{\prime} C \\
& =-T^{-1} C^{\prime} \Delta_{1}\left[\Delta_{1}^{-1} L \Delta_{1}^{-1}\left(\Delta_{1}^{-1}\right)^{\prime}+\Delta_{1}^{-1}\left(\Delta_{1}^{-1} L \Delta_{1}^{-1}\right)^{\prime}\right] \Delta_{1}^{\prime} C \\
& =-T^{-1} C^{\prime} \Delta_{1}\left[\Delta_{1}^{-1}\left(\Delta_{1}^{-1}\right)^{\prime}-\tau \tau^{\prime}\right] \Delta_{1}^{\prime} C=-T^{-1} I_{n},
\end{aligned}
$$

see also the proof of Theorem 1 in Marsh (2007a). Consequently and only when $\tau \in \mathcal{M}(X), \delta_{1, i}=T^{-1}$ and $\delta_{12, i}=T^{-1} \delta_{2, i}$. Substituting these into the definitions of $\gamma_{1}$ and $\gamma_{2}$ in the statement of Lemma 1 , we find

$$
Q_{1, n}(c)-Q_{0, n}(c)=0+\frac{c^{3}}{T}\left[\left(2 \sum_{i=1}^{n} \delta_{2, i} \tilde{\xi}_{2, i}^{2}-\sum_{i=1}^{n} \delta_{2, i} \tilde{\xi}_{12, i}^{2}\right)\right]+O_{p}\left(c^{4}\right) .
$$

Since also both $\sum_{i=1}^{n} \delta_{2, i} \tilde{\xi}_{2, i}^{2}$ and $\sum_{i=1}^{n} \delta_{2, i} \tilde{\xi}_{12, i}^{2}$ are $O_{p}(1)$ as $n \rightarrow \infty$, then we find $Q_{1}(c)-Q_{0}(c)=\lim _{n \rightarrow \infty}\left(Q_{1, n}(c)-Q_{0, n}(c)\right)=O_{p}\left(c^{4}\right)$, as required.

Part (ii). When $\tau \notin \mathcal{M}(X)$, put $k=1$ so $\bar{Q}=c^{-2}\left(Q_{1}(c)-Q_{0}(c)\right)$ is $O_{p}(1)$ and hence, immediately, $\Pi_{\alpha}(c)-\alpha=O\left(c^{2-\varpi_{1}}\right)$. However, when $\tau \in \mathcal{M}(X)$ put $k=2$ and $\bar{Q}=c^{-4}\left(Q_{1}(c)-Q_{0}(c)\right)$ is $O_{p}(1)$ so $\Pi_{\alpha}(c)-\alpha=O\left(c^{4-\varpi_{2}}\right)$ instead.

Part (iii). Since point optimal tests are unbiased and the power envelope is monotone, then for any $c \neq 0$ both $\Pi_{\alpha}^{\mu}(c)>\alpha$ and $\Pi_{\alpha}^{\tau}(c)>\alpha$. Suppose first 
that $c<0$, then the difference in rates implied by part (ii) implies there exists some value $c^{*}<0$ such that $\Pi_{\alpha}^{\tau}\left(c^{*}\right)<\Pi_{\alpha}^{\mu}\left(c^{*}\right)$.

Similar to the proof of Theorem 1 in Marsh (2011), let

$$
\begin{aligned}
F_{1, n}\left(\kappa_{\alpha}\right) & =\operatorname{Pr}\left[Q_{1, n}(c)<\kappa_{\alpha}\right]=\operatorname{Pr}\left[\left(\sum_{i=1}^{n} \lambda_{i} \tilde{z}_{i}^{2}\right)^{-1}<\kappa_{\alpha}\right]=\operatorname{Pr}\left[\sum_{i=1}^{n} \lambda_{i} \tilde{z}_{i}^{2}>\kappa_{\alpha}\right] \\
& =1-\frac{1}{2 \pi i} \int_{\tau-i \infty}^{\tau+i \infty} \frac{\exp \left\{n\left(R_{1}(\theta)-x \theta\right)\right\}}{\theta} d \theta
\end{aligned}
$$

where $R_{1}(\theta)=-\frac{1}{2 n} \sum_{i=1}^{n} \log \left(1-2 \theta \lambda_{i}\right)$ is the mean cumulant generating function of $\sum_{i=1}^{n} \lambda_{i} \tilde{z}_{i}^{2}$. Note that $\Pi_{\alpha}(c)=\lim _{n \rightarrow \infty} F_{1, n}\left(\kappa_{\alpha}\right)$, i.e. the asymptotic distributions are defined as the limit of the finite sample, consistent with the set-up of Lemma 1.

Since $A$ is an analytic function of $c$ then so are the $\lambda_{i}$ and hence so is $F_{1, n}\left(\kappa_{\alpha}\right)$, and its limit, through $R_{1}(\theta)$. Consequently both $\Pi_{\alpha}^{\mu}(c)$ and $\Pi_{\alpha}^{\mu}(c)$ are analytic in $c$, since they are functions only of the eigenvalues of $A$. Thus $\bar{\Pi}(c)=\Pi_{\alpha}^{\mu}(c)-\Pi_{\alpha}^{\tau}(c)$ is analytic in $c$. Note that $\bar{\Pi}(c) \geq 0$, since adding an additional invariance requirement - in this case for a linear trend - cannot increase power. Let $\mathbb{R}^{-}$denote the set of negative real numbers and let $U$ be any closed subset of $\mathbb{R}^{-}$. A fundamental property of bounded analytic functions is that if $\bar{\Pi}(c)=0$ for some $c \in U$ then $\bar{\Pi}(c)=0$ for all $c \in U$. Since this is not true for $c=c^{*}$ then it cannot be true for any $c$ satisfying $0>c>-\infty$, consequently it must be that $\bar{\Pi}(c)>0$ for all finite $c$. The proof for the case for $c>0$ is identical. 


\section{Supplementary Material}

The asymptotic power envelope of Elliott, Rothenberg and Stock (1996) was for-

mally derived under their Condition A, although can be readily generalized, as in the following Assumption taken from Chang and Park (2002). Let $\left(y_{t}\right)_{t=1}^{T}$ be generated from

$$
y_{t}=d_{t}+u_{t} \quad: \quad d_{t}=x_{t}^{\prime} \beta \quad \text { and } \quad u_{t}=\rho_{T} u_{t-1}+\varepsilon_{t},
$$

and assume:

Assumption 1 (i) $\left(\eta_{t}, \mathcal{F}_{t}\right)$ is a martingale difference sequence with filtration $\mathcal{F}_{t}$, and such that a) $\mathrm{E}\left[\eta_{t}^{2}\right]=\sigma^{2}$, b) $\operatorname{plim} T^{-1} \sum_{1 t}^{T \eta 2}=\sigma^{2}$, c) $\mathrm{E}\left[\left|\eta_{t}\right|^{s}\right] \leq m_{\varepsilon}<\infty$ for $s \geq 4$,

(ii) in (S1) $\varepsilon_{t}=\sum_{j=0}^{\infty} \psi_{j} \eta_{t-j}$, where $\sum_{j=0}^{\infty} j\left|\psi_{j}\right| \leq m_{\psi}<\infty, \psi_{0}=1$, and (iii) $u_{0}=o_{p}\left(T^{1 / 2}\right)$.

Denoting the power envelope by $\Pi_{\mu}(c)$ when $d_{t}=o\left(t^{1 / 2}\right)$, then

$$
\Pi_{\mu}(c)=\operatorname{Pr}\left[c^{2} \int_{0}^{1} W_{c}^{2}(t) d t-c W_{c}^{2}(1)<b_{\alpha}^{\mu}(c)\right]
$$

while when $d_{t}$ also includes a linear trend:

$$
\Pi_{\tau}(c)=\operatorname{Pr}\left[c^{2} \int_{0}^{1} V_{c}^{2}(t, c) d t-(1-c) V_{c}^{2}(1, c)<b_{\alpha}^{\tau}(c)\right] .
$$

In (S2) and $(\mathrm{S} 3) V_{c}(t, c)$ is the Gaussian process,

$$
V_{c}(t, c)=W_{c}(t)-t\left(\lambda W_{c}(1)-3(1-\lambda) \int_{0}^{t} s W_{c}(s) d s\right),
$$

with $\left.\lambda=(1-c) /\left(1-c+c^{2} / 3\right)\right), W_{c}(s)=\int_{0}^{s} e^{(r-s) c} d W(r), W(r)$ is standard Brownian motion and $b_{\alpha}^{\mu}(c)$ and $b_{\alpha}^{\tau}(c)$ are critical values chosen so that the point optimal tests have size $\alpha$. It is not apparent that $\Pi_{\tau}(c)<\Pi_{\mu}(c)$, unless these probabilities are numerically resolved.

\section{Proof of Lemma 1:}

(i) The asymptotic power envelope is the same for any process $\left\{y_{t}\right\}_{t \in \mathbb{Z}}$ constructed as in (S1) and satisfying Assumption 1. Therefore, without loss of any generality, we may assume that $\varepsilon_{t} \sim i i d N\left(0, \sigma^{2}\right), 0<\sigma<\infty$ and $u_{0}=0$. Let $y, X, \beta$ and $\Delta_{\rho}$ be as 
defined in the main text, and let $\varepsilon=\left(\varepsilon_{1}, . ., \varepsilon_{T}\right)$ then the equations in (S1) define the following generalized linear regression model, $y=X \beta+\Delta_{1+c / T}^{-1} \varepsilon$, or

$$
\Delta_{1} y=\Delta_{1} X \beta+\Delta_{1} \Delta_{1+c / T}^{-1} \varepsilon .
$$

Following the analysis of Marsh (2007a), put $W=\Delta_{1} X$ and apply the results of King (1980), then the maximal invariant for testing $H_{0}: c=0$ in (S4), is

$$
v=\frac{w}{|w|}=\frac{C^{\prime} \Delta_{1} y}{\sqrt{y^{\prime} \Delta_{1}^{\prime} M \Delta_{1} y}}, \quad M=I-W\left(W^{\prime} W\right)^{-1} W^{\prime},
$$

where $C C^{\prime}=M$ and $C^{\prime} C=I_{n}$. Also from King (1980), the density of $v$ (with respect to normalized Haar measure on the surface of the unit sphere in $n=T-k$ dimensions, $\left.\mathbb{S}^{n}\right)$ is

$$
p d f(v)=|A|^{-1 / 2}\left(v^{\prime} A^{-1} v\right)^{-(T-k) / 2},
$$

where $A=C^{\prime} \Delta_{1}^{\prime} \Delta_{\rho}^{-1}\left(\Delta_{\rho}^{-1}\right)^{\prime} \Delta_{1} C$.

Immediately, and see also Podivinsky and King (2000), the Neyman-Pearson point optimal test of $H_{0}: \rho=1$ vs. $H_{1}: \rho \neq 1$, is

$$
\text { reject } H_{0} \text { if } P_{v}=v^{\prime} A^{-1} v<\kappa_{\alpha} \text {, }
$$

where the critical value $\kappa_{\alpha}$ in (S5) is chosen so that the size of the test is $\alpha$.

Here the point optimal tests take the form of a quadratic form distributed on the sphere, rather than the difference in quadratic forms presented in Elliott, Rothenberg and Stock (1996). Marsh (2011) demonstrates the equivalency of these formulations using the marginal-likelihood methods developed in Francke and de Vos (2007), while Marsh (2007b) extends to the case of testing for a unit root in a lagged dependent variable.

Under $H_{0}: c=0, w=C^{\prime} \Delta_{1} y \sim N\left(0, \sigma^{2} I_{n}\right)$, so the critical value $k_{\alpha}$ of the the point optimal test (S5) for testing $H_{0}$ against $H_{1}: c \neq 0$, at size $\alpha$, is defined by

$$
\begin{aligned}
\alpha & =\operatorname{Pr}\left[v^{\prime} A^{-1} v<\kappa_{\alpha} \mid H_{0}\right]=\operatorname{Pr}\left[\frac{w^{\prime} A^{-1} w}{w^{\prime} w}<\kappa_{\alpha} \mid w \sim N\left(0, \sigma^{2} I_{n}\right)\right] \\
& =\operatorname{Pr}\left[\frac{\sum_{i=1}^{n} \lambda_{i}^{-1} z_{i}^{2}}{\sum_{i=1}^{n} z_{i}^{2}}<\kappa_{\alpha}\right],
\end{aligned}
$$

where $z_{i} \sim \operatorname{iidN}(0,1)$ and the $\left\{\lambda_{i}\right\}_{i=1}^{n}$ are the ordered eigenvalues of $A$. The power of (S5) against $H_{1}: c \neq 0$ is

$$
\Pi_{\alpha}(c)=\operatorname{Pr}\left[v^{\prime} A^{-1} v<\kappa_{\alpha} \mid H_{1}\right]=\operatorname{Pr}\left[\frac{w^{\prime} A^{-1} w}{w^{\prime} w}<\kappa_{\alpha} \mid w \sim N\left(0, \sigma^{2} A\right)\right] .
$$


Letting $z=\left(z_{1}, . ., z_{n}\right)^{\prime}=\sigma^{-1} A^{-1 / 2} w \sim N\left(0, I_{n}\right)$, then

$$
\Pi_{\alpha}(c)=\operatorname{Pr}\left[\frac{z^{\prime} z}{z^{\prime} A z}<\kappa_{\alpha}\right]=\operatorname{Pr}\left[\frac{\sum_{i=1}^{n} \lambda_{i}^{-1} z_{i}^{2}}{\sum_{i=1}^{n} z_{i}^{2}}<\kappa_{\alpha}\right],
$$

again with $z_{i} \sim \operatorname{iidN}(0,1)$. Defining $\bar{z}_{i}=z_{i} / \sqrt{\sum_{i=1}^{n} z_{i}^{2}}$ and then

$$
Q_{0, n}(c)=\sum_{i=1}^{n} \lambda_{i}^{-1} \bar{z}_{i}^{2} \text { and } Q_{0, n}(c)=\left(\sum_{i=1}^{n} \lambda_{i} \bar{z}_{i}^{2}\right)^{-1}
$$

establishes part (i) of the Lemma via the limit $n \rightarrow \infty$.

(ii) Define the two infinite sums,

$$
Q_{0}(c)=\lim _{n \rightarrow \infty} \sum_{i=1}^{n} \lambda_{i}^{-1} \tilde{z}_{i}^{2} \quad \text { and } \quad Q_{1}(c)=\lim _{n \rightarrow \infty} \frac{1}{\sum_{i=1}^{n} \lambda_{i} \tilde{z}_{i}^{2}},
$$

then since $0<\lambda_{i}<\infty$ both $Q_{0}(c)$ and $Q_{1}(c)$ are $O_{p}(1)$, and satisfy

$$
\min _{i}\left(\lambda_{i}^{-1}\right)<Q_{j}(c)<\max _{i}\left(\lambda_{i}^{-1}\right), j=1,2 .
$$

Because the power envelope is constructed, at each point, via probabilities involving both statistics a stochastic expansion the difference between $Q_{1}(c)$ (the random variable defining power) and $Q_{0}(c)$ (the random variable defining size) is required. When $c=0$, we have that $\lambda_{i}=1$ for all $i$, and so the problem is degenerate, with $Q_{1}(0)-Q_{0}(0)=0$, or local to the origin in $c$,

$$
Q_{1}(c)=Q_{0}(c)+o_{p}(1)
$$

For any finite $c, A$ and $A^{-1}$ are positive definite, symmetric and have eigenvalues and vectors satisfying, respectively,

$$
\lambda_{i} A=\lambda_{i} v_{i} \quad \text { and } \quad \lambda_{i}^{-1} A^{-1}=\lambda_{i}^{-1} v_{i}
$$

with $v_{i}^{\prime} v_{i}=1$. $A$ is analytic in $\rho$, and hence $c$, with derivatives

$$
\frac{d^{j} A}{d c^{j}}=C^{\prime} \Delta_{1}^{\prime} \frac{d^{j} \Sigma_{1+c / T}}{d c^{j}} \Delta_{1} C
$$

where $\Sigma_{\rho}=\left(T_{\rho}^{-1}\right)^{\prime} T_{\rho}$.

We can write down Taylor Series expansions around $c=0$ of both $Q_{0, n}(c)$ and $Q_{1, n}(c)$. First we have,

$$
Q_{0, n}(c)=\sum_{i=1}^{n} \lambda_{i}^{-1} \tilde{z}_{i}^{2}=1+\sum_{i=1}^{n}\left(\left.\sum_{j=1}^{\infty} \frac{c^{j}}{j !} \frac{d^{j} \lambda_{i}^{-1}}{d c^{j}}\right|_{c=0}\right) \tilde{z}_{i}^{2},
$$


while for $Q_{1, n}(c)$ we instead have,

$$
\begin{aligned}
Q_{1, n}(c) & =\frac{1}{\sum_{i=1}^{n} \lambda_{i} \tilde{z}_{i}^{2}}=\frac{1}{\left(1+\sum_{i=1}^{n}\left(\left.\sum_{j=1}^{\infty} \frac{c^{j}}{j !} \frac{d^{j} \lambda_{i}}{d c^{j}}\right|_{c=0}\right) \tilde{z}_{i}^{2}\right)} \\
& =1+\sum_{i=1}^{n}\left(\sum_{k=1}^{\infty}(-1)^{k}\left[\left.\sum_{j=1}^{\infty} \frac{c^{j}}{j !} \frac{d^{j} \lambda_{i}}{d c^{j}}\right|_{c=0} \tilde{z}_{i}^{2}\right]^{k}\right) .
\end{aligned}
$$

Including up to $O_{p}\left(c^{3}\right)$ terms, we then obtain

$Q_{0, n}(c)=1+\left.c \sum_{i=1}^{n} \frac{d \lambda_{i}^{-1}}{d c}\right|_{c=0} \tilde{z}_{i}^{2}+\left.\frac{c^{2}}{2} \sum_{i=1}^{n} \frac{d^{2} \lambda_{i}^{-1}}{d c^{2}}\right|_{c=0} \tilde{z}_{i}^{2}+\left.\frac{c^{3}}{6} \sum_{i=1}^{n} \frac{d^{3} \lambda_{i}^{-1}}{d c^{3}}\right|_{c=0} \tilde{z}_{i}^{2}+O_{p}\left(c^{4}\right)$,

and

$$
\begin{aligned}
Q_{1, n}(c)= & 1-\left.c \sum_{i=1}^{n} \frac{d \lambda_{i}}{d c}\right|_{c=0} \tilde{z}_{i}^{2}-\frac{c^{2}}{2}\left[\left.\sum_{i=1}^{n} \frac{d^{2} \lambda_{i}}{d c^{2}}\right|_{c=0} \tilde{z}_{i}^{2}-2\left(\left.\sum_{i=1}^{n} \frac{d \lambda_{i}}{d c}\right|_{c=0} \tilde{z}_{i}^{2}\right)^{2}\right] \\
& -\frac{c^{3}}{6}\left[6\left(\left.\sum_{i=1}^{n} \frac{d \lambda_{i}}{d c}\right|_{c=0} \tilde{z}_{i}^{2}\right)^{3}-3\left(\left.\sum_{i=1}^{n} \frac{d \lambda_{i}}{d c}\right|_{c=0} \tilde{z}_{i}^{2}\right)\left(\left.\sum_{i=1}^{n} \frac{d^{2} \lambda_{i}}{d c^{2}}\right|_{c=0} \tilde{z}_{i}^{2}\right)\right. \\
& \left.+\left.\sum_{i=1}^{n} \frac{d^{3} \lambda_{i}}{d c^{3}}\right|_{c=0} \tilde{z}_{i}^{2}\right]+O_{p}\left(c^{4}\right) .
\end{aligned}
$$

Considering first the $O_{p}(c)$ terms, notice that for all $i$,

$$
\frac{d \lambda_{i}}{d c}=v_{i}^{\prime} \frac{d A}{d c} v_{i} \quad \text { and } \quad \frac{d \lambda_{i}^{-1}}{d c}=v_{i}^{\prime} \frac{d A^{-1}}{d c} v_{i}
$$

see Section 8.7 of Magnus and Neudecker (1988). We then have

$$
\left.\frac{d \lambda_{i}^{-1}}{d c}\right|_{c=0}=\left.v_{i}^{\prime} \frac{d A^{-1}}{d c} v_{i}\right|_{c=0}=-\left.v_{i}^{\prime} A^{-1} \frac{d A}{d c} A^{-1} v_{i}\right|_{c=0}=-\left.\frac{d \lambda_{i}}{d c}\right|_{c=0},
$$

since $\left.A\right|_{c=0}=I_{n}$, and $\frac{d A^{-1}}{d c}=-A^{-1} \frac{d A}{d c} A^{-1}$. Immediately we then find that $Q_{1, n}(c)-$ $Q_{0, n}(c)=O_{p}\left(c^{2}\right)$, for all cases.

For the $O_{p}\left(c^{2}\right)$ and $O_{p}\left(c^{3}\right)$ terms, although in principle expressions for the higherorder derivatives of the eigenvalues and their inverse can be found resolving them can prove prohibitive since they also involve the derivatives of the eigenvectors. Instead, for $Q_{1, n}(c)$, write

$$
\begin{aligned}
\left.\sum_{i=1}^{n} \frac{d^{j} \lambda_{i}}{d c^{j}}\right|_{c=0} \tilde{z}_{i}^{2} & =\left.\frac{d^{j} \sum_{i=1}^{n} \lambda_{i} \tilde{z}_{i}^{2}}{d c^{j}}\right|_{c=0}=\left.\frac{d^{j} \tilde{z}^{\prime} A \tilde{z}}{d c^{j}}\right|_{c=0} \\
& =\left.\tilde{z}^{\prime} \frac{d^{j} A}{d c^{j}} \tilde{z}\right|_{c=0}=\left.\tilde{z}^{\prime} D_{j} \tilde{z}\right|_{c=0},
\end{aligned}
$$


where $\tilde{z}=z / \sqrt{\sum_{i=1}^{n} z_{i}^{2}}$ and $D_{j}$ is defined in the statement of the theorem.

Now let $\left\{\delta_{j, i}, v_{j, i}\right\}_{i=1}^{n}$ be the eigenvalues and eigenvectors of $D_{j}$ and define

$$
E_{j}=\left(\tilde{\xi}_{j, 1}, . ., \tilde{\xi}_{j, n}\right)^{\prime}=U_{j} \tilde{z}
$$

where $U_{j}=\left\{v_{j, 1}, . ., v_{j, n}\right\}$, so that we can write

$$
\begin{aligned}
Q_{1, n}(c)= & 1-c \sum_{i=1}^{n} \delta_{1, i} \tilde{\xi}_{1, i}^{2}+\frac{c^{2}}{2}\left[2\left(\sum_{i=1}^{n} \delta_{1, i} \tilde{\xi}_{1, i}^{2}\right)^{2}-\sum_{i=1}^{n} \delta_{2, i} \tilde{\xi}_{2, i}^{2}\right] \\
& -\frac{c^{3}}{6}\left[6\left(\sum_{i=1}^{n} \delta_{1, i} \tilde{\xi}_{1, i}^{2}\right)^{3}-3\left(\sum_{i=1}^{n} \delta_{1, i} \tilde{\xi}_{1, i}^{2}\right)\left(\sum_{i=1}^{n} \delta_{2, i} \tilde{\xi}_{2, i}^{2}\right)+\sum_{i=1}^{n} \delta_{3, i} \tilde{\xi}_{3, i}^{2}\right] \\
& +O_{p}\left(c^{4}\right) .
\end{aligned}
$$

For $Q_{0, n}(c)$, and similar to (S6), we also have

$$
\left.\sum_{i=1}^{n} \frac{d^{j} \lambda_{i}^{-1}}{d c^{j}}\right|_{c=0} \tilde{\xi}_{i}^{2}=\left.\tilde{z}^{\prime} \frac{d^{j} A^{-1}}{d c^{j}}\right|_{c=0} \tilde{z},
$$

so that if we successively differentiate the identity $A^{-1} A=I$ (noting that all derivatives of $A$ are symmetric) then we obtain,

$$
\begin{aligned}
\frac{d A^{-1}}{d c} A+\frac{d A}{d c} A^{-1} & =0, \\
\frac{d^{2} A^{-1}}{d c^{2}} A+2 \frac{d A^{-1}}{d c} \frac{d A}{d c}+A^{-1} \frac{d^{2} A}{d c^{2}} & =0, \\
\frac{d^{3} A^{-1}}{d c^{3}} A+3 \frac{d^{2} A^{-1}}{d c^{2}} \frac{d A}{d c}+3 \frac{d A^{-1}}{d c} \frac{d^{2} A}{d c^{2}}+A^{-1} \frac{d^{3} A}{d c^{3}} & =0 .
\end{aligned}
$$

Evaluating these derivatives at $c=0$ and noting the definition of $D_{j}$, then we find

$$
\begin{aligned}
\left.\frac{d A^{-1}}{d c}\right|_{c=0} & =-D_{1} \\
\left.\frac{d^{2} A^{-1}}{d c^{2}}\right|_{c=0} & =2\left(D_{1}\right)^{2}-D_{2} \\
\left.\frac{d^{3} A^{-1}}{d c^{3}}\right|_{c=0} & =-6\left(D_{1}\right)^{3}+6 D_{1} D_{2}-D_{3} .
\end{aligned}
$$

As a consequence, and letting $\left\{\delta_{12, i}, v_{12, i}\right\}_{i=1}^{n}$ be the eigenvalues and eigenvectors of the matrix $D_{1} D_{2}$, we then have the following stochastic expansion for $Q_{0, n}(c)$,

$$
\begin{aligned}
Q_{0, n}(c)= & 1-c \sum_{i=1}^{n} \delta_{1, i} \tilde{\xi}_{1, i}^{2}+\frac{c^{2}}{2} \sum_{i=1}^{n}\left(2 \delta_{1, i}^{2} \tilde{\xi}_{1, i}^{2}-\delta_{2, i} \tilde{\xi}_{2, i}^{2}\right) \\
& +\frac{c^{3}}{6} \sum_{i=1}^{n}\left(-6 \delta_{1, i}^{3} \tilde{\xi}_{1, i}^{2}+6 \delta_{12, i} \tilde{\xi}_{12, i}^{2}-\delta_{3, i} \tilde{\xi}_{3, i}^{2}\right)+O_{p}\left(c^{4}\right),
\end{aligned}
$$


where the $\tilde{\xi}_{12, i}$ are elements of the vector $E_{12}=U_{12} \tilde{z}$ and $U_{12}=\left\{v_{12,1}, . ., v_{12, n}\right\}$.

Directly subtracting (S7) from (S8) we get

$$
\begin{aligned}
Q_{1, n}(c)-Q_{0, n}(c)= & c^{2}\left[\left(\sum_{i=1}^{n} \delta_{1, i} \tilde{\xi}_{1, i}^{2}\right)^{2}-\sum_{i=1}^{n} \delta_{1, i}^{2} \tilde{\xi}_{1, i}^{2}\right] \\
& +c^{3}\left[\sum_{i=1}^{n} \delta_{1, i}^{3} \tilde{\xi}_{1, i}-\left(\sum_{i=1}^{n} \delta_{1, i} \tilde{\xi}_{1, i}^{2}\right)^{3}+\right. \\
& \left.\frac{1}{2}\left(\sum_{i=1}^{n} \delta_{1, i} \tilde{\xi}_{1, i}^{2}\right)\left(\sum_{i=1}^{n} \delta_{2, i} \tilde{\xi}_{2, i}^{2}\right)-\sum_{i=1}^{n} \delta_{12, i} \tilde{\xi}_{12, i}^{2}\right] \\
& +O_{p}\left(c^{4}\right) .
\end{aligned}
$$

Taking the limit as $n \rightarrow \infty$ of (S9) with

$$
\sqrt{n} \tilde{\xi}_{1, i}^{2} \rightarrow_{p} \xi_{1, i} \sim i i d N(0,1), \quad \text { for } i=1, \ldots, n
$$

since $n^{-1} \sum_{i=1}^{n} z_{i}^{2} \rightarrow_{p} 1$, and analogous results for $\tilde{\xi}_{2, i}^{2}$ and $\tilde{\xi}_{12, i}^{2}$ then establishes part (ii) of the Lemma.

\section{Proof of Lemma 2:}

For brevity put $Q_{0}=Q_{0}(c)$ and $Q_{1}=Q_{1}(c)$ and let $\bar{Q}=c^{-2 k}\left[Q_{0}-Q_{1}\right]$. Using the characterization of the asymptotic power envelope derived in Lemma 1, we have

$$
\begin{aligned}
\Pi_{\alpha}(c) & =\operatorname{Pr}\left[Q_{1}<\kappa_{\alpha}\right]=\operatorname{Pr}\left[Q_{0}+\left(Q_{0}-Q_{1}\right)<\kappa_{\alpha}\right]=\operatorname{Pr}\left[Q_{0}-c^{2 k} \bar{Q}<\kappa_{\alpha}\right] \\
& =\operatorname{Pr}\left[\bar{Q}>c^{-2 k}\left(Q_{0}-k_{\alpha}\right)\right] .
\end{aligned}
$$

Now let $f\left(q_{0}, \bar{q}\right)$ denote the joint density of $\left(Q_{0}, \bar{Q}\right)$ and let $f_{Q_{0}}\left(q_{0}\right)$ and $f_{\bar{Q}}(\bar{q})$ denote the marginal densities of $Q_{0}$ and $\bar{Q}$, so that

$$
\begin{aligned}
\Pi_{\alpha}(c)= & \int_{0}^{\infty} \int_{c^{-2 k}\left(q_{0}-\kappa_{\alpha}\right)}^{\infty} f\left(q_{0}, \bar{q}\right) d \bar{q} d q_{0} \\
& \left(\int_{0}^{\kappa_{\alpha}}+\int_{\kappa_{\alpha}}^{\kappa_{\alpha}+\varepsilon}+\int_{\kappa_{\alpha}+\varepsilon}^{\infty}\right)\left(\int_{c^{-2 k}\left(q_{0}-\kappa_{\alpha}\right)}^{\infty} f\left(q_{0}, \bar{q}\right) d \bar{q}\right) d q_{0} .
\end{aligned}
$$

If we take the three (double) integrals in (S10) individually, then we first have

$$
\int_{0}^{\kappa_{\alpha}} \int_{c^{-k}\left(q_{0}-\kappa_{\alpha}\right)}^{\infty} f\left(q_{0}, \bar{q}\right) d \bar{q} d q_{0} \leq \int_{0}^{\kappa_{\alpha}} \int_{-\infty}^{\infty} f\left(q_{0}, \bar{q}\right) d \bar{q} d q_{0}=\int_{0}^{\kappa_{\alpha}} f_{Q_{0}}\left(q_{0}\right) d q_{0}=\alpha .
$$


The second integral is,

$$
\begin{aligned}
\int_{\kappa_{\alpha}}^{\kappa_{\alpha}+\varepsilon} \int_{c^{-k}\left(q_{0}-\kappa_{\alpha}\right)}^{\infty} f\left(q_{0}, \bar{q}\right) d \bar{q} d q_{0} & \leq \int_{\kappa_{\alpha}}^{\kappa_{\alpha}+\varepsilon} f_{Q_{0}}\left(q_{0}\right) d q_{0} \\
& \leq \varepsilon\left(\sup _{\kappa_{\alpha} \leq q_{0} \leq \kappa_{\alpha}+\varepsilon} f_{Q_{0}}\left(q_{0}\right)\right) \leq \varepsilon M_{1}<\infty,
\end{aligned}
$$

the latter following since $Q_{0}$ is (the limit of) a quadratic form on the unit sphere with finite density, e.g. see Hillier (2001). The final integral satisfies,

$$
\begin{aligned}
\int_{\kappa_{\alpha}+\varepsilon}^{\infty} \int_{c^{-2 k}\left(q_{0}-\kappa_{\alpha}\right)}^{\infty} f\left(q_{0}, \bar{q}\right) d \bar{q} d q_{0} & \leq \int_{\kappa_{\alpha}+\varepsilon}^{\infty} \int_{c^{-2 k} \varepsilon}^{\infty} f\left(q_{0}, \bar{q}\right) d \bar{q} d q_{0} \leq \int_{c^{-2 k} \varepsilon}^{\infty} f\left(q_{0}, \bar{q}\right) d \bar{q} \\
& =\operatorname{Pr}\left[\bar{Q}>c^{-2 k} \varepsilon\right] \leq \operatorname{Pr}\left[|\bar{Q}|>c^{-2 k} \varepsilon\right] \\
& =\operatorname{Pr}\left[e^{2 \eta k|\bar{Q}|} \geq e^{2 \eta k c^{-2 k} \varepsilon}\right] \\
& \leq \frac{E\left[e^{2 \eta k|\bar{Q}|}\right]}{e^{2 \eta k c^{-2 k} \varepsilon}}=\frac{M_{\eta}(2 k|\bar{Q}|)}{e^{2 \eta k c^{-2 k} \varepsilon}}
\end{aligned}
$$

where $M_{\eta}(2 k|\bar{Q}|)$ is the moment generating function of $2 k|\bar{Q}|$. Both $Q_{1}$ and $Q_{2}$ are bounded above by $\max _{i \in \mathbb{Z}} \lambda_{i}^{-1}$ and below by $\min _{i \in \mathbb{N}} \lambda_{i}^{-1}$. Since $\lambda_{i}$ is continuous and differentiable on $[0, c]$ then so also are $\max _{i \in \mathbb{N}} \lambda_{i}^{-1}$ and $\min _{i \in \mathbb{N}} \lambda_{i}^{-1}$, both therefore permit a mean value expansion and are bounded on $[0, c]$. Consequently, $|\bar{Q}|$ is bounded, hence $M_{\eta}(2 k|\bar{Q}|) \leq M_{2}<\infty$. Adding (S11), (S12) and (S13) we have,

$$
\Pi_{\alpha}(c) \leq \alpha+\varepsilon M_{1}+\frac{M_{2}}{e^{2 \eta k c^{-2 k} \varepsilon}} .
$$

and following the proof of Lemma 3 in Bykhovskaya and Phillips (2017), we can put $\varepsilon=-c^{k}(\ln c)>0$, giving

$$
\Pi_{\alpha}(c) \leq \alpha+O\left(c^{2 k-\varpi_{k}}\right)
$$

for some, arbitrarily small, $\varpi_{k}>0$, as required. 


\section{Tables}

The following tables report the outcomes of experiments detailed in the discussion and conclusion of the main paper. All reported values are based on simulations involving 100000 Monte Carlo replications.

Table 1a: Power Envelopes, with a constant and various trends, for testing $H_{0}: c=0$ vs. $H_{1}^{S}$ and $H_{1}^{E}$ in model $(\mathrm{S} 1), T=250$.

\begin{tabular}{c|cccccc}
\multicolumn{1}{c|}{$d_{t}$} & $\beta_{1}$ & $\beta_{1}+\beta_{2} \ln t$ & $\beta_{1}+\beta_{2} \sqrt{t}$ & $\beta_{1}+\beta_{2} t$ & $\beta_{1}+\beta_{2} t^{2}$ & $\beta_{1}+\beta_{2} e^{t}$ \\
$c$ & & & & & & \\
\hline 1.25 & 0.361 & 0.351 & 0.332 & 0.057 & 0.076 & 0.356 \\
1.00 & 0.274 & 0.262 & 0.246 & 0.052 & 0.074 & 0.269 \\
0.75 & 0.197 & 0.188 & 0.181 & 0.051 & 0.067 & 0.194 \\
0.50 & 0.130 & 0.125 & 0.127 & 0.050 & 0.065 & 0.129 \\
0.25 & 0.085 & 0.079 & 0.083 & 0.050 & 0.054 & 0.084 \\
\hline-1.0 & 0.079 & 0.066 & 0.055 & 0.052 & 0.063 & 0.078 \\
-2.0 & 0.121 & 0.083 & 0.065 & 0.061 & 0.076 & 0.117 \\
-3.0 & 0.172 & 0.114 & 0.082 & 0.074 & 0.084 & 0.168 \\
-4.0 & 0.233 & 0.134 & 0.097 & 0.092 & 0.108 & 0.225 \\
-5.0 & 0.294 & 0.156 & 0.122 & 0.113 & 0.141 & 0.286
\end{tabular}


Table 1b: Approximations to $O_{p}\left(c^{3}\right)$ from Lemma 1 of the Power Envelopes

\begin{tabular}{c|cccccc}
\multicolumn{7}{c}{$H_{0}: c=0 \quad$ vs. $H_{1}^{S}$ and $H_{1}^{E}$ in model $(\mathrm{S} 1), T=250}$. \\
\multicolumn{1}{c|}{$d_{t}$} & $\beta_{1}$ & $\beta_{1}+\beta_{2} \ln t$ & $\beta_{1}+\beta_{2} \sqrt{t}$ & $\beta_{1}+\beta_{2} t$ & $\beta_{1}+\beta_{2} t^{2}$ & $\beta_{1}+\beta_{2} e^{t}$ \\
\hline 1.25 & 0.331 & 0.306 & 0.309 & 0.052 & 0.074 & 0.313 \\
1.00 & 0.246 & 0.230 & 0.237 & 0.052 & 0.072 & 0.241 \\
0.75 & 0.184 & 0.168 & 0.183 & 0.051 & 0.066 & 0.181 \\
0.50 & 0.128 & 0.121 & 0.121 & 0.050 & 0.061 & 0.127 \\
0.25 & 0.083 & 0.076 & 0.080 & 0.050 & 0.053 & 0.083 \\
\hline-1.0 & 0.077 & 0.057 & 0.057 & 0.050 & 0.053 & 0.076 \\
-2.0 & 0.118 & 0.063 & 0.067 & 0.051 & 0.058 & 0.127 \\
-3.0 & 0.183 & 0.077 & 0.101 & 0.051 & 0.061 & 0.178 \\
-4.0 & 0.244 & 0.114 & 0.128 & 0.053 & 0.074 & 0.255 \\
-5.0 & 0.332 & 0.185 & 0.203 & 0.056 & 0.099 & 0.307
\end{tabular}

Table 2: Ratio of the trend and constant Power Envelopes

for testing $H_{0}: c=0$ vs. $H_{1}^{S}$ and $H_{1}^{E}$ in model (S1).

\begin{tabular}{cc|ccc|ccc|ccc} 
& $\alpha$ & 0.01 & 0.01 & 0.01 & 0.05 & 0.05 & 0.05 & 0.10 & 0.10 & 0.10 \\
\hline$c$ & $T$ & 50 & 250 & 500 & 50 & 250 & 500 & 50 & 250 & 500 \\
\hline 1.25 & & 0.063 & 0.060 & 0.054 & 0.161 & 0.158 & 0.156 & 0.254 & 0.241 & 0.248 \\
1.00 & & 0.080 & 0.071 & 0.068 & 0.191 & 0.188 & 0.183 & 0.286 & 0.285 & 0.283 \\
0.75 & & 0.141 & 0.128 & 0.110 & 0.273 & 0.261 & 0.254 & 0.371 & 0.368 & 0.360 \\
0.50 & 0.262 & 0.221 & 0.212 & 0.405 & 0.386 & 0.381 & 0.502 & 0.485 & 0.484 \\
0.25 & & 0.515 & 0.459 & 0.422 & 0.622 & 0.581 & 0.580 & 0.697 & 0.668 & 0.663 \\
\hline-1.0 & 0.394 & 0.319 & 0.315 & 0.440 & 0.371 & 0.362 & 0.478 & 0.421 & 0.409 \\
-2.0 & 0.425 & 0.352 & 0.347 & 0.448 & 0.390 & 0.381 & 0.484 & 0.427 & 0.417 \\
-3.0 & 0.455 & 0.409 & 0.396 & 0.479 & 0.434 & 0.436 & 0.501 & 0.466 & 0.457 \\
-4.0 & 0.503 & 0.521 & 0.502 & 0.531 & 0.550 & 0.506 & 0.553 & 0.529 & 0.527 \\
-5.0 & 0.655 & 0.648 & 0.632 & 0.677 & 0.689 & 0.694 & 0.689 & 0.672 & 0.672
\end{tabular}


Table 3: Ratio of the trend and constant Power Envelopes for testing $H_{0}: c=0$ vs. $H_{1}^{F}$ in model (S1).

\begin{tabular}{cc|ccc|ccc|ccc} 
& $\alpha$ & 0.01 & 0.01 & 0.01 & 0.05 & 0.05 & 0.05 & 0.10 & 0.10 & 0.10 \\
\hline$c$ & $T$ & 50 & 250 & 500 & 50 & 250 & 500 & 50 & 250 & 500 \\
\hline 1.25 & & 0.125 & 0.114 & 0.101 & 0.247 & 0.246 & 0.235 & 0.351 & 0.345 & 0.343 \\
1.00 & & 0.183 & 0.162 & 0.140 & 0.320 & 0.306 & 0.287 & 0.422 & 0.411 & 0.393 \\
0.75 & 0.260 & 0.233 & 0.219 & 0.407 & 0.378 & 0.382 & 0.504 & 0.477 & 0.485 \\
0.50 & 0.382 & 0.346 & 0.337 & 0.535 & 0.514 & 0.500 & 0.622 & 0.605 & 0.594 \\
0.25 & & 0.627 & 0.590 & 0.606 & 0.738 & 0.704 & 0.712 & 0.781 & 0.776 & 0.781 \\
\hline-1.0 & 0.787 & 0.797 & 0.796 & 0.765 & 0.768 & 0.774 & 0.740 & 0.778 & 0.783 \\
-2.0 & 0.613 & 0.621 & 0.625 & 0.596 & 0.639 & 0.634 & 0.586 & 0.630 & 0.630 \\
-3.0 & 0.544 & 0.541 & 0.545 & 0.537 & 0.525 & 0.526 & 0.511 & 0.521 & 0.524 \\
-4.0 & 0.455 & 0.468 & 0.472 & 0.488 & 0.474 & 0.468 & 0.454 & 0.465 & 0.459 \\
-5.0 & 0.411 & 0.419 & 0.426 & 0.428 & 0.408 & 0.415 & 0.440 & 0.405 & 0.407
\end{tabular}

\section{Additional References}

Bykhovskaya, A. and P.C.B. Phillips 2017. Point optimal testing with roots that are functionally local to unity. Cowles Foundation discussion paper, $300 \%$.

Chang, Y. and J.Y. Park 2002. On the asymptotics of ADF tests for unit roots. Econometric Reviews 21: 431-447.

Hillier, G. 2001. The Density of a Quadratic Form in a Vector Uniformly Distributed on the n-Sphere. Econometric Theory, 17: 1-28.

Magnus, J.R. and Neudecker, H. 1999. Matrix Differential Calculus, with Applications in Statistics and Econometrics. Chichester: Wiley.

Marsh, P. 2007b. Constructing Optimal tests on a Lagged dependent variable. J.T.S.A. 28: 723-743.

Podivinsky, J. and M.L. King 2000. The exact power envelope of tests for a unit root. Discussion Papers in Economics and Econometrics: University of Southampton. 